\title{
RFInD: An RFID-based System to Manage Virtual Spaces
}

\author{
Akhilesh Saxena, Samrat Ganguly, Sudeept Bhatnagar, and Rauf Izmailov \\ NEC Laboratories America, \\ Princeton, NJ, USA. \\ Email: \{saxena,samrat,sudeept,rauf\}@nec-labs.com
}

\section{INTRODUCTION}

We present RFInD, a cost-effective utilitarian system for locating objects using RFID $[1,2]$ technology. RFInD separates the notion of location from that of physical coordinates by using the abstraction of a Virtual Space. A virtual space is created by using RFID tags to label entities and locations in the physical space as references. RFInD manages the virtual space by using the references to create a spatial map, over which objects can be tracked and located.

\section{RFInD}

RFInD is designed to achieve the following objectives: 1) Creation or a virtual space, 2) Embedding objects in the virtual space, and 3) Navigation of the virtual space to reach the target objects. RFInD aims to identify the physical space with reference tags affixed on static objects (e.g. desk, closet). The objects to be located are denoted as Target objects which are assumed to be mostly moveable (e.g. book). Target objects are affixed with object tags. RFInD uses each reference tag to identify a Containment Space. A collection of containment space defines the Virtual Space. The target objects embedded in this virtual space are associated with one or more containment spaces. When a user wishes to locate an object, RFInD can navigate her through the virtual space to the object's container. For example, if a reference tag identifies a desk in a room and an object tag identifying a book is associated with it, we can recognize the book is contained in the desk. Thus, RFInD does not have an explicit notion of exact physical coordinates. Instead, RFInD provides a notion of space containment to locate target objects.

\subsection{Prototype}

Figure 1 illustrates our RFInD prototype. A computer connected to the RFID reader allows us to control the reader's gain settings and communicate with RFInD database machine through its wireless interface (using XML, with SOAP as the underlying protocol). A GUI at the same computer allows users to manage and navigate the virtual space. The RFInD database maintains two tables to store the information required for system operation: 1)The Virtual Space Table containing information regarding all the reference tags in the system and 2) The Object Tag Table consisting of the details for each new inserted object in the system. For efficient user navigation, our prototype requires prior con-

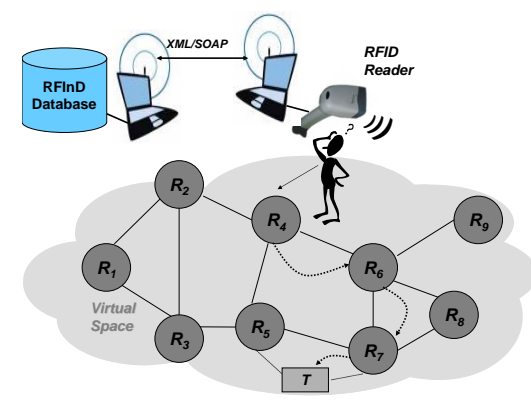

Figure 1: RFInD Prototype.

struction of the virtual space and embedding of target objects in it. For virtual space creation, reference tags are set up at various positions and the associate set for each tag is found. XML messages are constructed with each of the tag's id, its corresponding location (e.g., "Shelf"), its physical/logical associates and sent to the RFInD database to populate the Virtual Space Table. Relatively moveable objects (e.g. "Book") are embedded in the virtual space by finding the associate set for the object tag, adding the description of the object to form an XML message, and sending this message to the RFInD database to update the Object Tag Table. Our current navigation model lets the user specify a tag-id or description of a object which she wants to reach. The system first finds the closest tag $S$ from the current location of the user. Using the database, a graph is constructed from association sets of all tags and the path from $S$ to the destination is found. Figure 2 shows RFInD's ability to locate an object by creating and managing virtual spaces across an office space.

\subsection{Primitive Operations}

In providing the desired functionalities, we first carefully characterized a commercially available RFID reader. The reader's API allows us to control the gain of the transmitted signal. Our extensive experiments show: 1) For a given gain, the maximum angle at which a tag can be reliably read reduces with a increasing distance between the tag and the antennae, 2) Inter-tag interference reduces the probability of reading a tag, and 3) Different gain and phase values can be used to distinguish between tags' relative locations. Based on the above characterization, RFInD implements the following operations: 


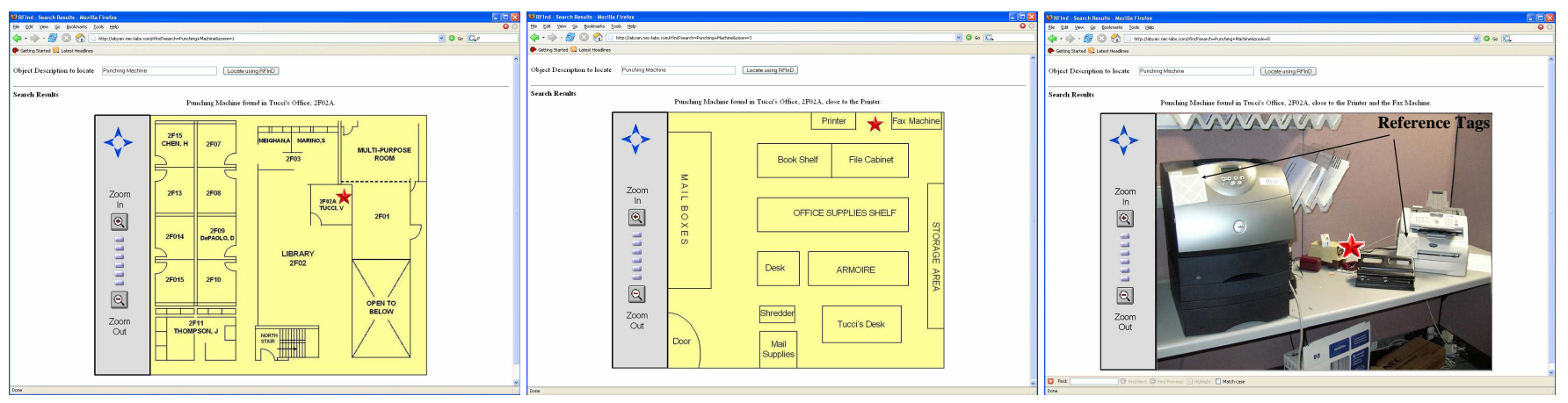

Figure 2: Using RFInD to locate a Punching Machine over a virtual space mapped to the Office's floor plan. Zoom levels show the object being located close to a Printer and a Fax Machine.

1)Proximity Detection: The proximity operation returns the id of the closest tag to the current location of the reader. A binary search is performed on the reader's gain values until the minimum gain value $\left(g_{\min }\right)$ is reached at which a set of tags can be read $\left(T_{\text {min }}\right)$. At $g_{\text {min }}$, the reader's antennae are rotated to find if some other tags can be read at any other angle (these tags could already be in $\left.T_{m i n}\right)$. If at some phase such tags are found, the binary search is resumed from the reader's minimum gain value to a maximum of $g_{\text {min }}$. If some tags are read at a gain $g^{\prime}$ less than $g_{\min }$ at some phase, $g_{\min }$ is set to $g^{\prime}$ and a new $T_{\text {min }}$ is created for these tags. Table

Table 1: Read Probability with Correct Association

\begin{tabular}{|l|l|l|l|}
\hline gain (\%) & Closer Tag & Object Tag & Farther Tag \\
\hline 6 & 1 & 0.3 & 0 \\
\hline 8 & 1 & 1 & 0 \\
\hline 22 & 1 & 1 & 0.7 \\
\hline
\end{tabular}

1 shows the read probabilities of identifying the Farther and Closer reference tags placed at 5 and 15 feet respectively, along with the Object tag placed at 8 feet from the reader. Using 0.5 as the threshold probability to read a tag, we see that the three tags are identified at gain levels of $6 \%, 8 \%$ and $22 \%$. Using square root of the gains as the notion for distances, we see that the object tag is considered close to the Closer tag.

Table 2: Read Probability with Skewed Association

\begin{tabular}{|l|l|l|l|}
\hline gain (\%) & Closer Tag & Object Tag & Farther Tag \\
\hline 6 & 1 & 0 & 0 \\
\hline 16 & 1 & 0.9 & 0 \\
\hline 25 & 1 & 1 & 0.3 \\
\hline 28 & 1 & 1 & 1 \\
\hline
\end{tabular}

2)Tag Association: This operation is used to find a set of reference tags around a target $\operatorname{tag} T$, to which $T$ can be linked with in the virtual space. To approximate the position of $T$, the area is scanned at the maximum gain of the reader. The approximate straight line at which $T$ resides is estimated by identifying the phase at which $T$ can be read at minimum gain $\left(g_{\min }\right)$. Possibly at $g_{\min }$, a large number of tags can be read $\left(T^{\prime}\right)$ along with $T$.
While reducing the gain in steps, the unread tags from $T^{\prime}$ are eliminated and added to $T_{a s s o c}$ until $T_{a s s o c}$ has some members. Lastly, the reader's antennae are rotated in both directions away from the straight line to $T$ in steps of 10 degrees at $g_{\min }$ and $g_{\min }+1$ until $T$ is not read or some new tags that weren't visible during the previous steps are found. These tags are added to $T_{\text {assoc }}$ to obtain the associate set of $T$. With the same setup as in the previous operation, we moved the object tag to 11 feet from the reader at a phase of 10 degrees. Table 2 shows the read probability, where the object tag is incorrectly associated with the Closer tag. To remedy this, we use Tag Clustering by placing a bunch of tags close to the Farther tag. As shown in Table 3, the object tag is correctly associated with the Farther tag.

Table 3: Impact of Clustering on Association

\begin{tabular}{|l|l|l|l|}
\hline gain (\%) & Closer Tag & Object Tag & Either of Farther Tags \\
\hline 6 & 1 & 0 & 0 \\
\hline 16 & 1 & 0.9 & 0 \\
\hline 18 & 1 & 0.9 & 0 \\
\hline 20 & 1 & 1 & 0.8 \\
\hline
\end{tabular}

\section{CONCLUSION}

We presented the design of the RFInD system that uses a single mobile RFID reader to create a Virtual Space by using reference tags to label entities and locations. Experiences with our prototype indicate that RFInD can be put to varied uses such as locating lost objects, managing objects in hospitals and libraries, and navigating the visually impaired.

\section{REFERENCES}

[1] S. Manapure, H. Darabi, V. Patel and P. Banerjee, "A comparative study of Radio Frequency-based Indoor Location Sensing Systems," In Proceedings of IEEE ICNSC, (March, 2004).

[2] P. De, K. Basu, and S. K. Das, "An Ubiquitous Architectural Framework and Protocol for Object Tracking using RFID Tags," In Proceedings of Mobiquitous, (August, 2004). 